\title{
Nanotechnology and Food Science: Tomorrow Design the Food
}

\author{
Shilpi Verma ${ }^{1 *}$ and Shobhana Gupta ${ }^{2}$ \\ ${ }^{1}$ Krishi Vigyan Kendra, Neemuch, India \\ ${ }^{2}$ Deputy Director Extension, RVSKVV, Gwalior, India \\ *Corresponding author
}

\begin{tabular}{|c|c|}
\hline & A B S T R A C T \\
\hline \multicolumn{2}{|l|}{ Keywords } \\
\hline $\begin{array}{l}\text { Food and } \\
\text { nanotechnology, } \\
\text { Nanotechnology, } \\
\text { Nanotechnology } \\
\text { application. }\end{array}$ & \multirow{3}{*}{$\begin{array}{l}\text { The paper deals with nanotechnology having an impact on several aspects } \\
\text { of food science, from how food is grown to how it is packaged. Companies } \\
\text { are developing nanomaterials that will make a difference not only in the } \\
\text { taste of food, but also in food safety, and the health benefits that food } \\
\text { delivers. }\end{array}$} \\
\hline Article Info & \\
\hline $\begin{array}{l}\text { Accepted: } \\
\text { 10 July } 2017 \\
\text { Available Online: } \\
\text { 10 September } 2017\end{array}$ & \\
\hline
\end{tabular}

\section{Introduction}

In today's competitive market technology is essential to keep leadership in the food and food processing industry. Consumers demand fresh authentic, convenient and flavorful food products. The future belongs to new products and new processes, with the goal of enhancing the performance of the product, prolonging the product shelf life and freshness, and improving the safety and quality of food.

Nanotechnology exhibits great potential for the food industry. It is an enabling technology that has the potential to revolutionize the food industry (Shefer, 2005). The potential of nanotechnology to revolutionize the health care, textile, materials, information and communication technology, and energy sectors has been well-publicized. In fact several products enabled by nanotechnology are already in the market, such as antibacterial dressings, transparent sunscreen lotions, stainresistant fabrics, scratch free paints for cars, and self cleaning windows. Nanotechnology is having an impact on several aspects of food science, from how food is grown to how it is packaged. Companies are developing nanomaterials that will make a difference not only in the taste of food, but also in food safety, and the health benefits that food delivers. The application of nanotechnology to the agricultural and food industries was first addressed by a United States Department of Agriculture roadmap published in 
September 2003 (Joseph and Morrison, 2006). The prediction is that nanotechnology will transform the entire food industry, changing the way food is produced, processed, packaged, transported, and consumed. The anticipated impact of nanotechnology upon society is frequently referred to as the "second industrial revolution" (Wernette, 2009). Rapid advances in nanotechnology promise to revolutionize many of the ways we manufacture products, produce energy, diagnose and treat diseases and increase global food production.

\section{What is nanotechnology?}

Nanotechnology is defined as the study and use of structures between 1 nanometer and 100 nanometers in size. Nanotechnology is the manipulation or self-assembly of individual atoms, molecules, or molecular clusters into structures to create materials and devices with new or vastly different properties. The definition of nanotechnology is based on the prefix "nano" which is from the Greek word meaning "dwarf". In more technical terms, the word "nano" means $10^{-9}$, or one billionth of something.

\section{How is nanotechnology being used in food science?}

The term 'nanofood' describes food which has been cultivated, produced, processed or packaged using nanotechnology techniques or tools, or to which manufactured nanomaterials have been added (Joseph and Morrison 2006). Examples of nanoingredients and manufactured nanomaterial additives include nanoparticles of iron or zinc, and nanocapsules containing ingredients like co-enzyme Q10 or Omega 3.

Because nanotechnology is essentially a set of techniques that allow manipulation of properties at a very small scale, it can have many applications:
- Canola oil contains tiny nano-particles that block cholesterol from entering the bloodstream.

- A chocolate shake is tastier and more nutritious thanks to the addition of nanosized ingredients 100,000 times smaller than a grain of sand.

- Food packages are embedded with nanoparticles that alert consumers when a product is no longer safe to eat.

- Nanomaterials enhance the biological activity of dietary supplements or nutraceuticals by allowing delivery of nutrients directly into cells.

Experts estimate nanotechnology will be incorporated into $\$ 20$ billion worth of consumer food products by 2010 as the world's largest food companies use the super small to make big improvements in food packaging, safety and nutrition (Wernette, 2009). The Helmut Kaiser Consultancy Group, a pro-nanotechnology analyst, suggests that there are now over 300 nano food products available on the market worldwide. It estimates that the global nano food market was worth US\$5.3 billion in 2005 and will rise to US $\$ 20.4$ billion by 2010. It predicts that nanotechnology will be used in $40 \%$ of the food industries by 2015 (Miller, 2008)

\section{Food science: Current nanotechnology applications}

There are four key focus areas for nanotechnology food research:

-Nano-modification of seed and fertilizers/ pesticides

-Food 'fortification' and modification -Interactive 'smart' food

•'Smart' packaging and food tracking 


\section{Food 'fortification' and modification}

Nanotech companies are working to fortify processed food with nano-encapsulated nutrients, its appearance and taste boosted by nano-developed colors, its fat and sugar content removed or disabled by nanomodification, and 'mouth feel' improved. Food 'fortification' will be used to increase the nutritional claims that can be made about a given processed food - for example the inclusion of 'medically beneficial' nanocapsules will soon enable chocolate chip cookies or hot chips to be marketed as health promoting or artery cleansing. Nanotechnology will also enable junk foods like ice cream and chocolate to be modified to reduce the amount of fats and sugars that the body can absorb. This could happen either by replacing some of the fats and sugars with other substances, or by using nanoparticles to prevent the body from digesting or absorbing these components of the food. In this way, the nano industry could market vitamin and fibrefortified, fat and sugar-blocked junk food as health promoting and weight reducing (Miller, 2008).

\section{Nanotechnology in food microbiology}

Detection of very small amounts of a chemical contaminant, virus or bacteria in food systems is another potential application of nanotechnology. The exciting possibility of combining biology and nanoscale technology into sensors holds the potential of increased sensitivity and therefore a significantly reduced response-time to sense potential problems. Nanosensors that are being developed by researchers at both Purdue and Clemson universities use nanoparticles, which can either be tailor-made to fluoresce different colors or, alternatively, be manufactured out of magnetic materials. These nanoparticles can then selectively attach themselves to any number of food pathogens. Employees, using handheld sensors employing either infrared light or magnetic materials, could then note the presence of even minuscule traces of harmful pathogens. The advantage of such a system is that literally hundreds and potentially thousands of nanoparticles can be placed on a single nanosensor to rapidly, accurately and affordably detect the presence of any number of different bacteria and pathogens. A second advantage of nanosensors is that, given their small size, they can gain access into the tiny crevices where the pathogens often hide (Shefer, 2005)

The application of nanotechnologies on the detection of pathogenic organisms in food and the development of nanosensors for food safety is also studied at the Bioanalytical Microsystems and Biosensors Laboratory at Cornell University. The focus of the research performed at Cornell University is on the development of rapid and portable biosensors for the detection of pathogens in the environment, food and for clinical diagnostics. The bioanalytical microsystems use the same biological principles as were used in the simple biosensors, i.e. RNA recognition via DNA/RNA hybridization and liposome amplification. The bioanalytical microsystems that are studied focus on the very rapid detection of pathogens in routine drinking water testing, food analysis, environmental water testing and in clinical diagnostics (Shefer, 2005)

Nanosensors are being developed that can detect bacteria and other contaminates, such as salmonella, at a packaging plant. This will allow for frequent testing at a much lower cost than sending samples to a lab for analysis. This point-of-packaging testing, if conducted properly, has the potential to dramatically reduce the chance of contaminated food reaching grocery store shelves. 
Nanotechnology in food packaging and safety

Nanotechnology offers many possibilities here. Hollow nanofibres and silicate nanoparticles or metallic/ceramic nanoparticles (carbon nanofibres) combine light weight and strength. These new materials are currently routinely produced in laboratories and can be used for both equipment and structures.

Another promising application in food packaging is Gas-barrier structures with low permeability. Nanoparticles embedded in plastics may be used to retard the diffusion of gases such as oxygen or water vapor by creating tortuous paths. Such products are already on the market.

Encoding or decoding individual surfaces, counterfeit protection using nanotaggants, nanocrystaline indicators to sense and signal modified atmosphere environments within packages, light-activated oxygen sensing links, food deterioration sensors and power for smart packaging, such as radiofrequency identification (RFID). Nanotubes may be filled with materials such as antimicrobials to disperse in the plastic matrix. Materials that could improve bioactivity and thereby biodegradability of polyesters like PLA. Surfaces can be nanostructured in order to influence their adhesiveness to products or dirt and there by their susceptibility to fouling, which is important in food processing and food packaging.

Clay nanocomposites are being used to provide an impermeable barrier to gasses such as oxygen or carbon dioxide in lightweight bottles, cartons and packaging films. Storage bins are being produced with silver nanoparticles embedded in the plastic. The silver nanoparticles kill bacteria from any material that was previously stored in the bins, minimizing health risks from harmful bacteria (www.understandingnano.com/food. html, 2008) Researchers are using silicate nanoparticles to provide a barrier to gasses (for example oxygen), or moisture in a plastic film used for packaging. This could reduce the possibly of food spoiling or drying out. Zinc oxide nanoparticles can be incorporated into plastic packaging to block UV rays and provide anti bacterial protection, while improving the strength and stability of the plastic film. Researchers are also working on pesticides encapsulated in nanoparticles; that only release pesticide within an insect's stomach, minimizing the contamination of plants themselves.

\section{Interactive 'smart' food}

Companies such as Kraft and Nestlé are designing 'smart' foods that will interact with consumers to 'personalize' food, changing colour, flavor or nutrients on demand. Kraft is developing a clear tasteless drink that contains hundreds of flavors in latent nanocapsules. A domestic microwave could be used to trigger release of the colour, flavor, concentration and texture of the individual's choice. Nanocapsules that contain flavor or color enhancers are embedded in the food; inert until a hungry consumer triggers them. 'Smart' foods could also sense when an individual was allergic to a food's ingredients, and block the offending ingredient. Or alternatively, 'smart' packaging could release a dose of additional nutrients to those which it identifies as having special dietary needs, for example calcium molecules to people suffering from osteoporosis (Miller, 2008)

\section{'Smart' packaging and food tracking}

Nanotechnology will dramatically extend food shelf life. Mars Inc. already has a patent on an invisible, edible, nano wrapper which will envelope foods, preventing gas and 
moisture exchange. 'Smart' packaging (containing nano-sensors and anti-microbial activators) is being developed that will be capable of detecting food spoilage and releasing nano-anti-microbes to extend food shelf life, enabling supermarkets to keep food for even greater periods before its sale. Nanosensors, embedded into food products as tiny chips that were invisible to the human eye, would also act as electronic barcodes. They would emit a signal that would allow food, including fresh food, to be tracked from paddock to factory to supermarket and beyond (Miller, 2008)

\section{Nanotechnology in food processing}

Principal applications are on demand preservatives and interactive foods. Nanocapsules can be incorporated into food to deliver nutrients. Addition of nanoparticles to existing food can enable increased absorption of nutrients. Applications are already being tested in new products to enter the market. Another key application is additives which could easily be absorbed by the body and could increase product shelf life. Nanosized dispersions, emulsions and filled micelles have the advantage that they are not subjected to sedimentation which gives better product life span and storage. As their size is much smaller than the wavelength of light, they can be incorporated in clear and transparent foods without causing muddiness. Substances difficult to dissolve can more easily be absorbed by the body if they are of nanoscale size due to their large surface area. If the active substance is to be protected during storage or passage through the intestines the existing nanotechnology can produce perfect protective layers. It is also possible to tailor protective layers to release active substances in an "intelligent" way, e.g. caused by a change of $\mathrm{pH}$-value.

In addition to packaging, nanotechnology is already making an impact on the development of functional or interactive foods, which respond to the body's requirements and can deliver nutrients more efficiently. Various research groups are also working to develop new "on demand" foods, which will remain dormant in the body and deliver nutrients to cells when needed. A key element in this sector is the development of nanocapsules that can be incorporated into food to deliver nutrients. Other developments in food processing include the addition of nanoparticles to existing foods to enable increased absorption of nutrients.

One of the leading bakeries in Western Australia has been successful in incorporating nanocapsules containing tuna fish oil (a source of omega 3 fatty acids) in their top selling product "Tip-Top" Up bread. The microcapsules are designed to break open only when they have reached the stomach, thus avoiding the unpleasant taste of the fish oil (Joseph and Morrison, 2006)

The Israeli Company Nutralease utilizes Nano-sized Self-assembled Liquid Structures (NSSL) technology to deliver nutrients in nanosized particles to cells. The particles are expanded micelles (hollow spheres made from fats, with an aqueous interior) with a diameter of approximately $30 \mathrm{~nm}$ (Garti, 2005). The nutrients or "nutraceuticals" are contained within the aqueous interior. Nutraceuticals that have been incorporated in the carriers include lycopene, beta-carotene, lutein, phytosterols, CoQ10 and DHA/EPA. The Nutralease particles allow these compounds to enter the bloodstream from the gut more easily, thus increasing their bioavailability. The technology has already been adopted and marketed by Shemen Industries to deliver Canola Activa oil, which it claims reduces cholesterol intake into the body by $14 \%$, by competing for bile solubilisation. This technology also has potential applications in the pharmaceutical industry. 
A number of chemical companies are researching additives which are easily absorbed by the body and can increase product shelf life. Biodelivery Sciences International have developed nanocochleates, which are $50 \mathrm{~nm}$ coiled nanoparticles and can be used to deliver nutrients such as vitamins, lycopene, and omega fatty acids more efficiently to cells, without affecting the colour or taste of food (www.biodeliveryscience.com,2010). Kraft foods have established a consortium of research groups from 15 universities to look into the applications of nanotechnology to produce interactive foods. These will allow the consumer to choose between different flavors and colors.

The consortium also has plans to develop smart foods which will release nutrients in response to deficiencies detected by nanosensors, and nanocapsules which will be ingested with food, but remain dormant until activated. All these new developments will make the concept of super foodstuffs a reality and these are expected to offer many different potential benefits including increased energy, improved cognitive functions, better immune function, and antiaging benefits.

Nanotechnology has already been used in the cosmetics industry to produce transparent creams. Royal Body Care, a company utilizing nanotechnology in nutritional sciences, has marketed a new product called NanoCeuticals which is a colloid (or emulsion) of particles of less than $5 \mathrm{~nm}$ in diameter. The company claims the product will scavenge free radicals, increase hydration and balance the body's $\mathrm{pH}$ (www.smartwoman.royalbodycare.com, 2005). The company has also developed NanoClustersTM, a nanosize powder combined with nutritional supplements. When consumed, it enhances the absorption of nutrients.
Food and Cosmetic Companies are working together to develop new mechanisms to deliver vitamins directly to the skin. For example, Nestlé, which has a $1 ` 49 \%$ stake in L'Oreal, is developing transparent sun creams to deliver vitamin $\mathrm{E}$ directly to skin. The aim is to manufacture a cream which is absorbed by the skin and releases Vitamin E slowly, in addition to providing UV protection. Transparent UV-blocking creams are already on the market and L'Oreal expects the cream with added functionality to be marketed soon. Other competitors such as Estée Lauder are manufacturing anti-ageing formulations that make use of nanoparticles.

The US based Oil fresh Corporation has marketed a new nanoceramic product which reduces oil use in restaurants and fast food shops by half. As a result of its large surface area, the product prevents the oxidation and agglomeration of fats in deep fat fryers, thus extending the useful life span of the oil. An additional benefit is that oil heats up more quickly, reducing the energy required for cooking (www.Oilfresh.com, 2010)

Wageningen University in Netherlands has recently established a research centre which will focus its research on the application of nanotechnology in the food industry. The Wageningen BioNT (Bionanotechnology) Centre will concentrate on various topics including: sensing and diagnostics of food quality and safety; encapsulation and delivery of nutrients; micro- and nanodevices for physical and (bio) chemical processing; chemical biology; nanotoxicology; and consumer science and technology assessment (BioNt, 2010).

The German company Aquanova has developed a new technology which combines two active substances for fat reduction and satiety into a single nano-carrier (micelles of average $30 \mathrm{~nm}$ diameters), an innovation said 
to be a new approach to intelligent weight management. Called NovaSOL Sustain, it uses CoQ1O to address fat reduction and alpha-lipoic acid for satiety. The NovaSol technology has also been used to create a vitamin $\mathrm{E}$ preparation that does not cloud liquids, called SoluE, and a vitamin C preparation called SoluC. The NovaSOL product can be used to introduce other dietary supplements as it protects contents from stomach acids (Aquanova, 2010)

In a different strategy, Unilever is developing low fat ice creams by decreasing the size of emulsion particles that give ice-cream its texture. By doing so it hopes to use up to $90 \%$ less of the emulsion and decrease fat content from $16 \%$ to about 1\% (Daily Telegraph, 2005)

The Woodrow Wilson International Center for Scholars in the US has produced a consumer database of marketed nanotechnology and has so far identified more than 15 items which have a direct relation to the food industry. The list includes nanoceuticals developed by RBC Life Sciences and Canola Activa oil developed by Shemen Industries; the use of silver nanoparticles in refrigerators manufactured by LG Electrical, Samsung and Daewoo to inhibit bacterial growth and eliminate odors; All Spray For Life ${ }^{\circledR}$ which is manufactured by Health Plus International and uses a newly-designed pre-metered, non-aerosol Nanoceautical Delivery System (NDS) for transmucosal administration of dietary supplements, resulting in increasedbioavailability compared with gastrointestinal absorption. A detailed list of products is available on the website (Nanotechproject, 2010)

\section{Nanotechnology in human nutrition}

Nanotechnology also promises to provide a means of altering and manipulating food products to more effectively and efficiently deliver nutrients, proteins and antioxidants to precisely target nutritional and health benefits to a specific site in the human body or to specific cells to enhance their efficacy and bioavailability. Several of the groups are studying the use of nanotechnology to encapsulate certain nutrients, flavors and colors and release them upon need or over an extended period of time. Functional food will benefit firstly from the new technologies, followed by normal food, nutraceuticals and others. Properties of nanoparticles may differ widely from 'conventional' forms. Growing scientific evidence indicates that:-

-Free nanoparticles can cross cellular barriers, and may reach those targets in the body where larger equivalents could have not reached

-Exposure to some ENPscan increase production of oxyradicals that may lead to oxidative damage and inflammatory reactions

-Potential benefits for industry and the consumer by maintaining quality and freshness, new tastes, flavors, textures, greater nutritional value, shelf life, better traceability and safety, less salt, sugar, fat and preservatives.

-Concerns over consumer safety lead to need for some basic research into potential health effects of nanofoods and for a vigilant self regulation/ best practice by the industry.

-Consumer information/ education are a must for the success of nanofoods.

Nanoparticles are being developed that will deliver vitamins or other nutrients in food and beverages without affecting the taste or appearance. These nanoparticles actually encapsulate the nutrients and carry them through the stomach into the bloodstream. Research is also being conducted to develop nanocapsules containing nutrients that would 
be released when nanosensors detect a vitamin deficiency in your body. Basically this research could result in a super vitamin storage system in your body that delivers the nutrients you need, when you need them.

In conclusion, major potential product applications for the nanosphere/microsphere system are baked goods, refrigerated/frozen dough and batters, tortillas and flat breads, processed meats, acidified dried meat products, microwavable entrees, seasoning blends, confectionery, specialty products, chewing gum, dessert mixes, nutritional foods and products for well-being, health bars, dry beverage mixes and many others.

Some companies are already aware of the impact of nanotechnology in the food industry. Research facilities are established, potential applications are under study, although only a handful of nano food products are now available in the market. Nevertheless, the tremendous potential will attract more and more competitors into this still untapped field.

The use of nano-surveillance in food packaging will also introduce new privacy concerns. As the food industry's use of nanotracking increases, it will gain the capacity to track the movement of food from the paddock, to the factory, to the supermarket and to your dinner plate. This will raise serious new privacy issues for which we are poorly prepared.

Nanotechnology can provide for the future development of far more precise and effective methods of, and other forms of, manipulation of food polymers and polymeric assemblages to provide tailor-made improvements to food quality and food safety. Nanotechnology promises not only the creation of novel and precisely defined material properties, it also promises that these materials will have selfassembling, self-healing and maintaining properties.

\section{References}

Daily Telegraph, (2005), How super cows and nanotechnology will make ice cream healthy, (21.8.05), cited in Joseph, Tiju and Morrison, Mark (2006), Nanotechnology in Agriculture and Food, a Nanoforum Report; www.nanoforum.org (accessed on 3/12/2010)

Garti, Nissin; (2005), www nutralease.com/technology.asp cited in Joseph, Tiju and Morrison, Mark (2006), Nanotechnology in Agriculture and Food, a Nanoforum Report; Www.nanoforum.org (accessed on 3/12/2010)

Joseph, Tiju and Morrison, Mark (2006), Nanotechnology in Agriculture and Food, a Nanoforum Report; www.nanoforum.org (accessed on 3/12/2010)

Miller, Georgia; (2008), Nanotechnology the new threat to food, www.globalresearch.ca/index.php?con text=va\&aid $=10755$ (accessed on $3 / 12 / 2010)$

Shefer,Adi;(2005), The Application of Nanotechnology in the Food Industry, Foodtech International, www.foodtech international.com/papers/applicationnano.htm - (accessed on 3/12/2010)

Wernette, Ronald;(2009), Nanotechnology Coming to your Store- Recall the hard lessons from GMO's and let's get it right this time, www.foodprocessing.com (accessed on $3 / 12 / 2010$ )

www.aquanova.de/product-micelle.html, (2010), cited in Joseph, Tiju and Morrison, Mark (2006), Nanotechnology in Agriculture and Food, a Nanoforum Report; www.nanoforum.org (accessed on 3/12/2010) 
www.biodeliveryscience.com/bioralnutrients. html (2010), cited in Joseph, Tiju and Morrison, Mark (2006), Nanotechnology in Agriculture and Food, a Nanoforum Report; www.nanoforum.org (accessed on $3 / 12 / 2010)$

www.biont.wur.nl/nl, (2010), cited in Joseph, Tiju and Morrison, Mark (2006), Nanotechnology in Agriculture and Food, a Nanoforum Report; www.nanoforum.org (accessed on $3 / 12 / 2010$ )

www.nanotechproject.org/index.php?id=44\&i $\mathrm{d}=44 \&$ action $=v i e w \& \mathrm{dbq}=$ food $\& \mathrm{p}=0$, (20100, cited in Joseph, Tiju and Morrison, Mark (2006), Nanotechnology in Agriculture and
Food, a Nanoforum Report; www.nanoforum.org (accessed on $3 / 12 / 2010$ )

www.oilfresh.com/of1000.html, (2010), cited in Joseph, Tiju and Morrison, Mark (2006), Nanotechnology in Agriculture and Food, a Nanoforum Report; www.nanoforum.org (accessed on 3/12/2010)

www.smartwoman.royalbodycare.com/nanote chnology_revolution.aspx, (2005), cited in Joseph, Tiju and Morrison, Mark (2006), Nanotechnology in Agriculture and Food, a Nanoforum Report; (accessed on 3/12/2010)

www.understandingnano.com/food.html, (2008) (accessed on 3/12/2010)

\section{How to cite this article:}

Shilpi Verma and Shobhana Gupta. 2017. Nanotechnology and Food Science: Tomorrow Design the Food. Int.J.Curr.Microbiol.App.Sci. 6(9): 3553-3561. doi: https://doi.org/10.20546/ijcmas.2017.609.437 